\section{Consummatory grooming fragments: A model for periodic behaviors*}

\author{
WALTER ROGERS, VIRGINIA PARSONS $\dagger$, and WALTER RANDALL \\ University of Iowa, Iowa City, Iowa 52240
}

Cats with midbrain lesions exhibit an abnormal dissociation between appetitive and consummatory grooming behaviors. Periodic regression analyses of two sets of behavioral data from groups studied in different years revealed a 4-month rhythm, with the same timing in both years. Various long-term endocrine rhythms that may be involved in the rhythmic disintegration of grooming behavior are discussed.

Randall (1964) described an abnormal grooming behavior which appears in cats following lesions of the caudal ventrolateral midbrain tegmentum: The delivery of light tactile stimuli to the body surface revealed a dissociation of the appetitive and consummatory grooming behaviors. In the normal cat the three grooming consummatory behaviors, licks, bites, and scratches, are preceded by orienting behaviors. In the lesioned cat the initial appetitive behaviors are missing and the consummatory responses are emitted directly as isolated fragments. Randall \& Parsons (1969) reported that the extent of the body surface from which these grooming fragments could be elicited showed rhythmic variations; a Fourier analysis revealed an approximate 4-month rhythm, with the dissociation most pronounced in June and October.

Periodic regression analysis of sunshine data from weather stations revealed the existence of 3 - to 5-month rhythms (Randall, 1970). Thus, it is conceivable that the rhythmic changes in sunshine may be the Zeitgeber for the periodic changes in grooming behavior. To check for such a possible relationship, a first step was an attempt to replicate the finding of a 4-month rhythm in the grooming abnormality of midbrain-lesioned cats.

*This research was supported by Grant MH 15402-03 from the Institute of Mental Health, U.S. Public Health Service. +Now at the University of Wisconsin-Parkside.
Then the climatological data for the time periods concurrent with the two sets of behavioral data were examined to determine if there was a significant correlation between behavior and sunshine.

\section{METHOD}

Six male cats with lesions similar to those illustrated by Randall (1964) were used. A quantitative measure of the areal extent of the dissociated grooming responses was made with the help of standard outline drawings of the left and right lateral views of a cat. Tactile stimuli were systematically directed to the body surface of the cat, and the areas from which grooming fragments could be elicited were plotted on the diagram. All cats were tested 26 times, once every 2 weeks for a year. The qualitative differences among licks, bites, and scratches were ignored; a total areal score was obtained for each cat and expressed as a percent of the body surface. These procedures were used in the previous study.

Sunshine data were obtained from the three nearest weather stations, Moline and Peoria in Illinois and Des Moines in Iowa. The arerage of these stations was assumed to be the best estimate of local sunshine conditions. Monthly percents of for August 1967 through July 1968 (the period of the previous study) and for February 1969 through January 1970 (the period of the present study). For convenience in comparison, all the data were arranged with measurements beginning in January, and Pearson product-moment correlations were obtained for the sunshine and behavioral data. possible sunshine scores were obtained
The two sets of behavioral data were fitted with the Fourier series,

$y_{t}=a_{0}+\sum_{i=1}^{j-1}\left(a_{i} \cos \frac{2 \pi i t}{k}+b_{i} \sin \frac{2 \pi i t}{k}\right)$,

where $y_{t}$ is the derived score for time $t, a_{0}$ is the grand mean, $a_{i}$ and $b_{i}$ are coefficients derived with least-squares criterion, $\mathrm{k}$ is the number of repeated measures, and $j$ is the last harmonic of the Fourier series $[j=$ $(\mathrm{k} / 2)]$. The analysis of variance developed by Bliss (1958) was used to evaluate the periodic regression: after least-square fits of the data were made, the sum of squares was partitioned into harmonic, harmonic interaction, remaining trend, and residual components. F tests involving remaining trend and residual error were used to truncate the Fourier series, and then $F$ tests with harmonic and harmonic interactions were used to evaluate the selected curve. Detailed examples of this procedure have been published (Randall, 1970).

\section{RESULTS}

The two groups of lesioned cats exhibited similar multiphasic variations in the area of the body surface from which grooming fragments were elicitable (Fig. 1). The two fitted curves show nearly simultaneous occurrences of troughs in April and August and peaks in June and October. The results of an analysis of variance (two-factor mixed design with repeated measures on one factor, Lindquist, 1953, p. 267) indicated that the two groups differed in overall level ( $\mathrm{F}=6.01, \mathrm{df}=1,15, \mathrm{p}<.01)$ but not in trend $(\mathrm{F}=1.28, \mathrm{df}=25,400$, $p>.10)$. The average percent of the

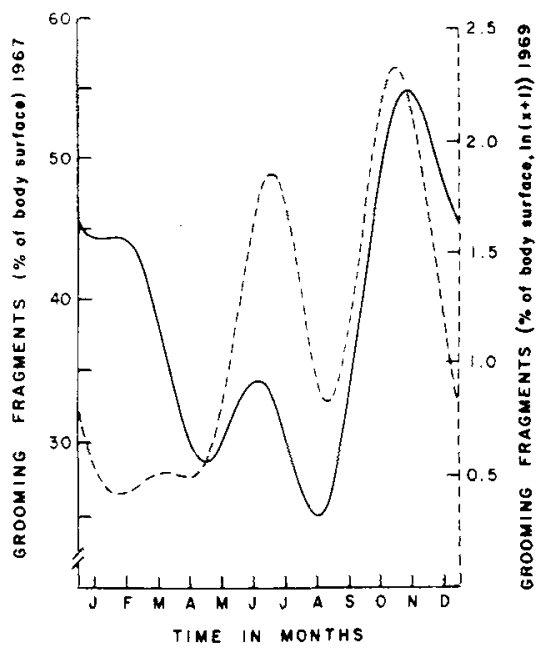

Fig. 1. The least-squares fitted curves to the areal grooming measures of two groups of lesioned cats. Twelve cats were in the 1967 study and six in the 1969 study. 
Table 1

Mean Squares and F Ratios from the Periodic Regression Analysis of Areal Grooming Scores

Areal Behavior Score

\begin{tabular}{|c|c|c|c|c|c|c|}
\hline \multirow{2}{*}{$\begin{array}{l}\text { Partitions of Total } \\
\text { Sum of Squares }\end{array}$} & \multicolumn{3}{|c|}{1967} & \multicolumn{3}{|c|}{1969} \\
\hline & MS & $\mathbf{D F}$ & $\mathbf{F}$ & MS & DF & $\mathbf{F}$ \\
\hline 1. Among Cats & 759,422 & 11 & $147.31 \dagger$ & 59.11 & 5 & $64.25+$ \\
\hline 2. Annual Harmonic $\left(a_{1} \& b_{1}\right)$ & 278,911 & 2 & $4.94^{*}$ & 38.95 & 2 & $7.04^{*}$ \\
\hline 3. Annual Harmonic Interaction & 56,418 & $\mathbf{2 2}$ & $10.94 t$ & 5.53 & 10 & $6.01 \div$ \\
\hline 4. 6-Month Harmonic $\left(a_{2} \& b_{2}\right)$ & 37,528 & $\mathbf{2}$ & 2.72 & 16.36 & 2 & $5.91 *$ \\
\hline 5. 6-Month Harmonic Interaction & 13,795 & 22 & $2.68 *$ & 2.77 & 10 & $3.01 *$ \\
\hline 6. 4-Month Harmonic $\left(a_{3} \& b_{3}\right)$ & 54,548 & 2 & $7.25 \dagger$ & 16.80 & 2 & $4.58 *$ \\
\hline 7. 4-Month Harmonic Interaction & $\mathbf{7 , 5 2 1}$ & 22 & 1.46 & 3.67 & 10 & $3.99 *$ \\
\hline 8. Remaining Trend & 7,407 & 19 & 1.44 & 1.60 & 19 & $1.74^{*}$ \\
\hline 9. Remainder (Random Error) & 5,155 & 209 & - & .92 & 95 & - \\
\hline
\end{tabular}

$* p<.05,+p<.01$

body surface yielding consummatory fragments was $39 \%$ in the 1967 group and $7 \%$ in the 1969 group. Histological examination revealed that the lesions of the 1969 group were smaller than those of the 1967 group, which probably accounts for the difference in overall level. The behavioral scores of the two groups were correlated; the Pearson product-moment correlation was $+.400(p<.05, \mathrm{df}=24)$.

The analysis of variance for the regression analyses of the curves of Fig. 1 are given in Table 1 . The data for 1967 were fitted with three harmonics (Rows 2, 4, and 6 in Table 1) and the trend sum of squares (Row 8 ) was reduced to the magnitude of the residual error (Row 9).

The 1969 data also were fitted with three harmonics, but the remaining trend (Row 8 ) was significantly greater than the residual error (Row 9). However, $91 \%$ of the trend was accounted for with the three harmonics. Many zero scores were present in the 1969 data during trough times (i.e., the cats reverted to a completely normal state on occasions), so that a $\ln (x+1)$ transformation was necessary to meet the assumptions of the statistical procedures.

The behavior scores were not correlated with sunshine, even though multiphasic variations in sunshine were found for the 2 years.

\section{DISCUSSION}

The results of the 1969 study of the appearance of grooming fragments in midbrain-lesioned cats are in substantial agreement with the results of the 1967 study. The two studies clearly establish the existence of a quantifiable rhythmic alteration in the grooming behavior of lesioned cats which occurs with consistent timing characteristics. Although the presence of rhythmic changes in the integration of grooming behavior is clear, the mechanisms involved are unknown. Earlier evidence (Randall \& Liittschwager, 1967) indicated that the behavioral rhythm was a concomitant of a 3- to 4-month rhythm in thyroid activity, but, although the abnormal grooming behaviors were found in thyroidectomized cats (Randall \& Parsons, 1970), thyroid hormone replacement therapy did not revert the behavioral state of lesioned cats to normal. Furthermore, no detectable differences were found in the thyroid hormone levels in the blood or brain of normal and midbrain-lesioned cats. And Rogers (1970), in a quantitative study, found a clear dissociation between thyroid activity and the abnormal grocming behavior. Thyroid activity, then, is not an aspect of the physiological bases of the lesion-induced dissociation between appetitive and consummatory grooming behaviors.

Long-term metabolic rhythms in adrenalin and noradrenalin (Parsons, 1970) and glucocorticoids (Randall \& Parsons $^{1}$ ) have been investigated. Although seasonal rhythms were found in each instance, the abnormal behavior was not a concomitant of any of the rhythms. Current research indicates that the lesion-induced grooming abnormality reverts temporarily to normal after systemic injections of 5 -hydroxytryptophan, suggesting that rhythmic fluctuations in serotonin may be involved in the genesis of the abnormal behavior. The general hypothesis that the timing of the behavioral dissociation is the result of the interaction of a damaged brain with rhythmic changes (either normally present or lesion induced) in the internal milieu as previously proposed (Randall et al, 1969) remains tenable.

The rhythmic synchrony in the abnormal dissociation of appetitive and consummatory grooming behaviors of the two groups of lesioned cats, studied in separate years, clearly specifies a problem in the interrelationships among external and internal factors in the integration of behavior and provides a unique model for the study of long-term periodic behaviors.
REFERENCES

BLISS, C. Periodic regression in biology and climatology. Bulletin 615, The Connecticut Agricultural Experimental Station, 1958.

LINDQUIST, E. Design and analysis of experiments in psychology and education. Boston: Houghton-Mifflin, 1953.

PARSONS, V. Long-term rhythms in grooming behavior, food intake, body weight, adrenalin, and noradrenalin in cats with lateral midbrain lesions. Unpublished PhD dissertation, University of Iowa, lowa City, Iowa, 1970.

RANDALL, W. The behavior of cats (Felis catus L.) with lesions in the caudal midbrain region. Behaviour, 1964, 23, 107-139.

RANDALL, W. Sunshine rhythms, a possible Zeitgeber for multiphasic biological rhy thms during a year. Journal of Interdisciplinary Cycle Research, 1970, 1, 389-404.

R ANDALL, W.. LAKSO, V., \& LIITTSCHWAGER, J. Lesion-induced dissociations between appetitive and consummatory behaviors and their relationship to body weight and food intake rhythms. Journal of Comparative \& Physiological Psychology, 1969, 68, 476-483.

RANDALL, W., \& LITTSCHWAGER, J. The relationship between cyclic changes in thyroid function and behavior of cats with brain stem lesions. Joumal of Psychiatric Research, 1967, 5, 39-58.

RANDALL, W., \& PARSONS, V. The concomitancy in the rhythms of caloric intake and behavior in cats: A replication. Psychonomic Science, 1969, 15, 35-36. RANDALL, W., \& PARSONS, V. Thyroidectomy produces abnormal grooming behavior in cats. Psychonomic Science, 1970, 21, 268-270.

ROGERS, W. Rhythmic variations during a year in grooming behavior. thyroid activity, and body weight of normal and midbrain-lesioned cats. Unpublished MA thesis, University of Iowa, Iowa City, Iowa, 1970.

UNITED STATES WEATHER BUREAU, Climatological Data, National Summary, U.S. Department of Commerce, Environmental Data Service, 1967-1970.

NOTE

1. Randall, W., \& Parsons, V. Rhythmic dysfunctions in 11-hydroxycorticoid excretion after midbrain lesions and their relationship to an abnormal grooming behavior in cats. Submitted for publication, 1971 . 